\title{
Optimized Access Network Selection in a Combined WLAN/LTE Environment
}

\author{
Karl Andersson · Christer Åhlund
}

\begin{abstract}
Multimode terminals equipped with multiple radio access technologies are becoming increasingly popular. At the same time, network operators and service providers seek opportunities to deliver seamless services cost effectively, leveraging a variety of radio access technologies using both licensed and unlicensed spectrum. In order to standardize the operations of such complex environments 3GPP is currently working on IP flow mobility and mobile data offload solutions. This article proposes and evaluates a new access network selection procedure in such a combined WLAN/LTE environment. The proposed solution takes not only parameters available in the mobile node and its current and candidate access networks into account, but performs an optimization on the heterogeneous wireless network level as well. An optimization model based on an approximate solution to the well-known bin packing problem is presented. Also, there is a signaling scheme for distribution handling presented. Results from simulations performed in OPNET Modeler show improvements compared to basing handover decisions on locally available information only.
\end{abstract}

Keywords: IP Flow Mobility, Mobile data offload, Optimized access network selection, Heterogeneous wireless networks, WLAN, LTE

K. Andersson

Pervasive and Mobile Computing Laboratory, Luleå University of Technology, SE-93187 Skellefteå, Sweden

Email: karl.andersson@1tu.se

C. Åhlund

Email: christer.ahlund@ltu.se 
The procedure of efficient and scalable access network selection is becoming an increasingly important feature of any heterogeneous wireless networking environment. This is the case because of the growing proportion of new handsets being equipped with more than one radio access technology and wireless access networks of various types being deployed widely with overlapping coverage areas.

There are a number of mobility support architectures for heterogeneous wireless networks proposed. One important aspect is at what layer in the communication stack mobility management is handled. Mobility management could be handled at the datalink layer for a single radio access technology. It might also be managed at the network layer [1][2] allowing mobile nodes to have their IP address fixed while roaming across subnets. Solutions at the transport [3] and application [4] layers also exist.

Other design aspects of mobility support solutions are whether the solution allows for simultaneous connections to multiple radio access networks and if handover decisions are host-based or network-based [5]. Yet other aspects are whether the mobile node is involved in mobility-related signaling and whether its IP communication stack remains unchanged or needs modification.

Standardization bodies like the IEEE, 3GPP and the IETF have been active introducing mobility support and access network selection mechanisms to their standards. IEEE released the Media independent handover services standard under the name of IEEE 802.21 [6] (figure 1), while 3GPP decided to add an entity named Access Network Discovery and Selection Function, ANDSF, to its architecture in Release 8 [7][8] (figure 2). IETF supports both of them with discovery mechanisms.

The scope of IEEE 802.21 covers discovery and selection of wireless access networks. These features are mainly handled by the IEEE 802.21 Information Service. The standard also covers handover negotiation and connectivity setup for the datalink and network layers handled by the IEEE 802.21 Event and Command Services. IEEE 802.21 allows both for host-based and network-based decision making. It should also be noted that the IEEE 802.21 Information Service allows both the mobile node to send requests to the server for certain information and also the server to push information to the mobile node. To support the new IEEE 802.21 standard, the IETF defined a framework for mobility support [9] and mechanisms for discovery of mobility services using DHCP options [10] and the DNS [11]. 


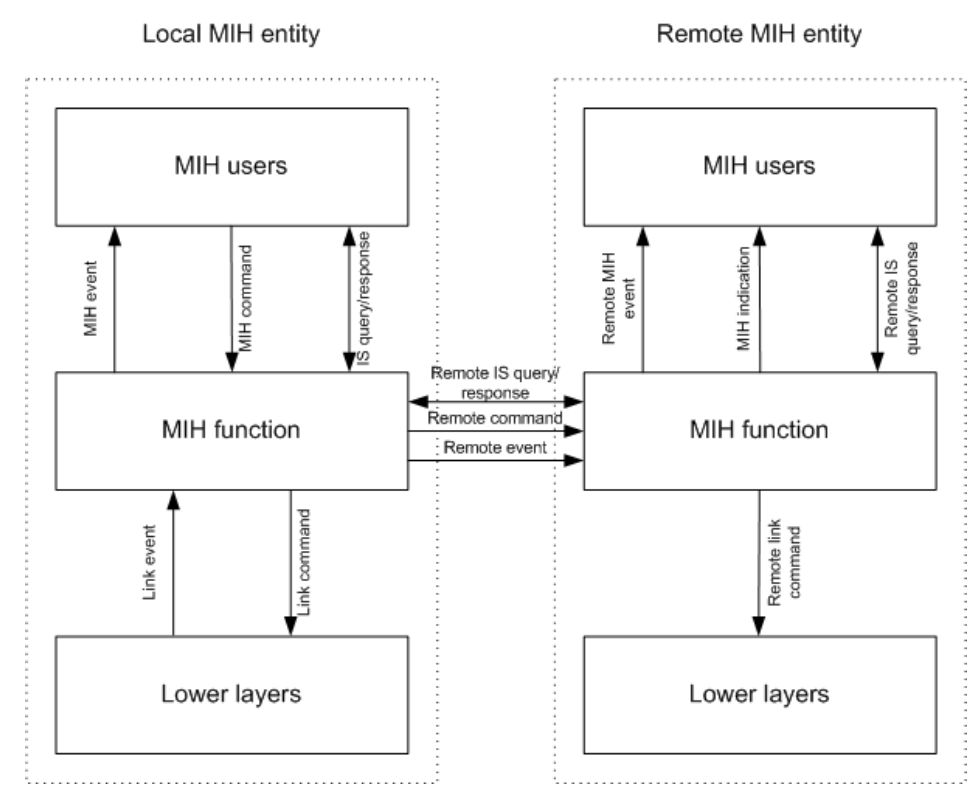

Fig. 1 Overall architecture of IEEE 802.21

The functionality provided by the 3GPP ANDSF entity covers data management and control functionality thus providing access network discovery and selection assistance to the mobile node. As for the case with the IEEE 802.21 Information Service, the ANDSF entity may both respond to requests from mobile nodes and push data to them. Information exchange is handled using the OMA Device Management Protocol [12] with the ANDSF Management Object being specified in [13]. Mobile nodes may connect to ANDSF entities both in the visited network and in their home networks. IETF is currently working on mechanisms for discovery of 3GPP ANDSF services using DHCP options [14].

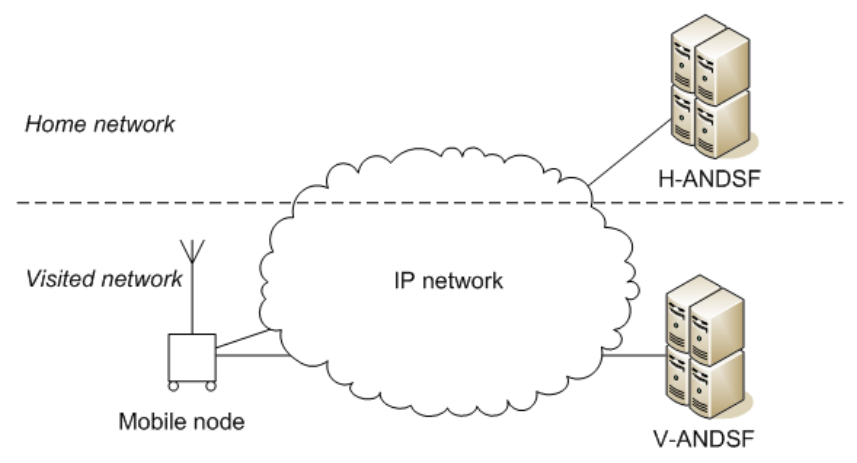

Fig. 2 Overall architecture of 3GPP ANDSF

Currently, 3GPP is also standardizing support for IP flow mobility and seamless WLAN offload [15] building the solution on Dual-stack Mobile IPv6 [16], multihoming [17], and flow-based mobility handling [18] using traffic selectors [19]. The goal is to provide mobile data offload support for LTE networks allowing multimode terminals to move certain flows to WLAN hotspots when overlapping coverage is available in the proximity of the terminal.

However, both the IEEE 802.21 standard and the functionality provided by the 3GPP standards only support mobile nodes with access network selection through delivery of operator-provided policies and static information about the topology of the infrastructure in the wireless access networks. Dynamic aspects such as fluctuations of network load are not handled at all.

This article proposes and evaluates a scheme for mobile nodes and their individual flows to perform access network selection. Flows are assigned to 
wireless access networks individually in a dynamic way reflecting terminal capabilities and network load. Overall performance in the heterogeneous wireless network, also referred to the mobility overlay network, is optimized.

The rest of the article is structured in the following way: Section 2 discusses related work, while Section 3 presents the proposed scheme for access network selection. Section 4 describes the optimization procedure, while simulations performed in OPNET Modeler are presented in Section 5. Section 6 presents the results achieved, while Section 7 finally concludes the article and indicates future work.

\section{Related Work}

There is already quite some work published in the area of optimized access network selection. McNair et al. [20] show that optimizing available network resources on the heterogeneous wireless network level outperforms decision making mechanisms based on only received signal strength or only using a cost function-based model in a local fashion.

Gazis et al. [21] model the Always Best Connected problem as a knapsack problem and argue that it is NP-hard. The realtime and distributed aspects of the proposed model are modeled in UML.

Song et al. [22] propose an access network selection scheme in an integrated WLAN and UMTS environment using mathematical modeling and computational techniques applying Analytic Hierarchy Process (AHP) to decide relative weights of various evaluation criterion and Grey Relational Analysis (GRA) to rank the network alternatives. Quality of Service is placed at the top of the AHP hierarchy while throughput, timeliness, reliability, security, and cost are at the second level in the AHP hierarchy. Received signal strength and coverage area are used to represent availability, while delay, response time, and jitter are used to represent timeliness. Finally bit error rate, burst error, and average number of retransmissions per packet define reliability.

Xing et al. [23] model the problem of access network selection as a variant of the bin packing problem. A number of approximation algorithms are proposed for finding near-optimal solutions. Performance was shown to gradually improve when using the proposed algorithms.

Finally, Haydar et al. [24] survey a number of algorithms for access network selection. Applying their proposal leads to a load balancing distribution between access networks.

In the area of mobility management solutions for heterogeneous wireless networks there has been a lot of related work published the past ten years. Gustafsson et al. [25] described the concept of being best connected and discussed the user experience and business relationships in an Always Best Connected environment.

Perera et al. [26] proposed a mobility toolbox architecture for All-IP networks including support for Mobile IPv4, Mobile IPv6, NEMO, and HIP. This coexistence is effected by means of a mobility toolbox enabling mobility handling to be selected according to context. The design of the toolbox is described as a component of the Ambient Networks architecture. Feasibility and performance gains are demonstrated with a prototype implementation of network mobility.

Kong et al. [27] analyzed both qualitatively and quantitatively networkbased approaches and host-based approaches to the mobility management problem. They stressed the key features of Proxy Mobile IPv6 and expected that it 
would be the mobility protocol of choice when realizing the next-generation AllIP mobile networks.

Moreover, Kafle et al. [28] presented mobility-related functions of locator ID separation-based network architectures. The functions include name resolution, handover optimization, and support for mobility across heterogeneous network protocols. Finally, Kiriyama et al. [29] proposed a simple context reflector for Proxy Mobile IPv6 enabling services like AAA (Authentication, Authorization, and Accounting), ROHC (Robust Header Compression) and QoS (Quality of Service) to be quickly re-established after handovers. Results were reported in terms of reduced handover latencies and packet loss during handovers were.

Performance studies on mobile data offload are so far very rare. However, Lee et al. [30] collected statistics for iPhone users and showed that users are covered by WiFi networks $70 \%$ of the time. On-the-spot WiFi offloading was reported to handle $60 \%$ of the traffic volume.

\section{$3 \quad$ Proposed Solution}

\subsection{Mobility Architecture}

The solution for access network selection being proposed in this article is built on previous work where a traffic load metric called the Relative Network Load, RNL, was defined [31] and an architecture for port-based multihomed Mobile IPv6 was proposed [32] (figure 3). Furthermore, service levels in that type of networking environment were proposed to be monitored using the model described in [33].

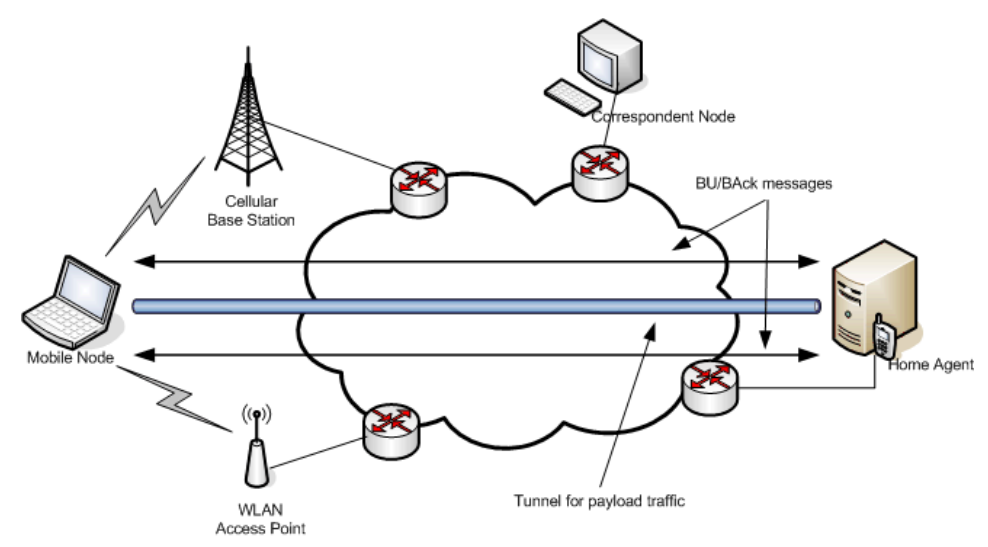

Fig. 3 Overall architecture of proposed solution

In the proposed architecture, the mobile nodes register their access network specific IP addresses (referred to as care of addresses, CoAs) with a centralized router, the home agent. Each mobile node is assigned an IP address in the subnet of the home agent (referred to as the home address, HoA). The HoA is used by higher layers as endpoint identifier for the mobile node. This address is fixed while the mobile node is roaming from one access network to another.

Registration messages are sent using Binding Update (BU) messages and acknowledged using Binding Acknowledgments (BAcks). The home agent holds a mapping table, referred to as the binding cache, where CoAs are mapped to HoAs and vice versa. Traffic is tunneled from the mobile node to the home agent, so that encapsulation and decapsulation is handled by the IP stack of the mobile node and the home agent.

Route optimization is covered by the Mobile IPv6 standard, allowing mobile nodes to send BU messages also to correspondent nodes. In this scenario, 
bidirectional tunnels are also set up to those correspondent nodes allowing traffic to bypass the home agent and be routed directly to any correspondent node supporting the Mobile IPv6 standard.

The RNL metric reflects the network load in a wireless access network and is computed using the BU messages and Backs of Mobile IPv6 also as probing packets measuring delay and jitter. The RNL metric is then calculated as follows:

$R N L_{n}=\bar{z}_{n}+c J_{n}$

$\bar{z}_{n}=\frac{1}{h} R T T_{n}+\frac{h-1}{h} \bar{z}_{n-1}$

$R T T_{n}=R_{n}-S_{n}$

$D_{n}=R_{n}-R_{n-1}-\left(S_{n}-S_{n-1}\right)=\left(R_{n}-S_{n}\right)-\left(R_{n-1}-S_{n-1}\right)=R T T_{n}-R T T_{n-1}$

$J_{n}=\frac{1}{h}\left|D_{n}\right|+\frac{h-1}{h} J_{n-1}$

where $S_{i}$ and $R_{i}$ are defined as

$S_{i}=$ the time of sending BU message $i$

$R_{i}=$ the time of arrival of BAck message $i$

$c$, and $h$ are positive, real constants. $h$ determines the history window for the weighted average calculations. $c$ determines the weight of the RTT in comparison to the RTT jitter value. The variables $\bar{z}, D$, and $J$ are initialized with the following values:

$\bar{z}_{0}=R T T_{0}$

$D_{0}=0$

$J_{0}=D_{1}$

In the multihomed version of Mobile IPv6 that is used in our architecture proposal, the BU messages are sent in parallel over all wireless access networks that the mobile node is covered by. This way an RNL value is computed for each available wireless access network in every mobile node.

\subsection{Access Network Selection Solution}

Our previous work based its handover decision making mechanism on RNL values and user-defined policies locally available in each mobile node. This article extends the decision making capability so that resources at the heterogeneous wireless network level are optimized.

In order to allow for the home agent to be aware of each mobile node's RNL values the format for the BU message header is proposed to be changed so that the RNL value for that specific wireless access network is sent embedded in the BU message header. Also, two flags were added in previous work, namely the $\mathrm{M}$ and $\mathrm{S}$ flags. The $\mathrm{M}$ flag indicates a multihomed binding while the $\mathrm{S}$ flag indicates that binding is to be regarded as the default binding (figure 4). 


\begin{tabular}{|c|c|c|c|}
\hline 8 bits & 8 bits & 8 bits & 8 bits \\
\hline Payload protc & Header len & MH Type & Reserved \\
\hline \multicolumn{2}{|c|}{ Checksum } & \multicolumn{2}{|c|}{ Sequence number } \\
\hline $\mathrm{A}|\mathrm{H}| \mathrm{L}|\mathrm{K}| \mathrm{M} \mid \mathrm{S}$ & RNL value & \multicolumn{2}{|c|}{ Lifetime } \\
\hline
\end{tabular}

Fig. 4 BU message header with $\mathrm{M}$ and $\mathrm{S}$ flags and room for RNL value

In our previous work, a flow mobility option was proposed to be added (figure 5). That option was introduced in order to allow the mobile node to signal any specific flow (indicated by its port and protocol) to be routed via the specific wireless access network that the BU message was sent over. Default routes were also proposed to be handled separately.

The solution being proposed in this article includes adding an $\mathrm{R}$ flag to the flow mobility option giving the mobile node an opportunity to indicate to the home agent to retain that binding regardless what is optimal on the heterogeneous wireless network level. This is useful if a flow in the mobile node has special requirements to be met regarding its preference on access network selection.

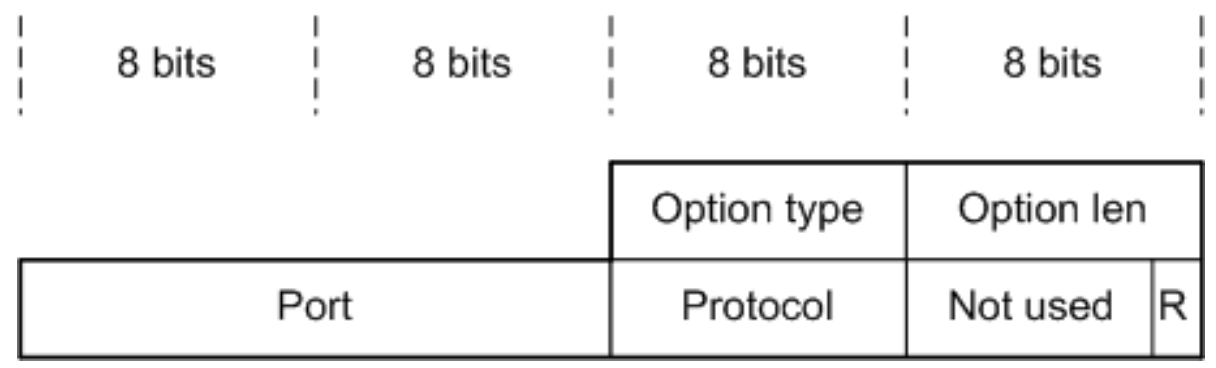

Fig. 5 Flow mobility option with R flag

An example of the binding cache in the home agent is depicted in Table 1. The binding cache is a mapping table used by the home agent for routing of traffic to the ultimate destination. The home address (HoA) is the fixed IP address used by higher layers as endpoint identification, while the care of address (CoA) indicates the topologically correct IP address reflecting the mobile node's current attachment to the Internet. Multihomed Mobile IPv6 allows for multiple bindings and the example show three bindings: one for TCP traffic to port 6935 , one for UDP traffic to port 7830, and a default binding (marked with "-1") where traffic should be routed if the protocol and port combination is not listed in the table. Lifetime indicates how long a particular binding should be kept and allows for soft state handling. In order to extend the lifetime of a binding, the mobile node may send a Binding Refresh Request (BRR) message.

Table 1 Example of binding cache

\begin{tabular}{|c|c|c|c|c|}
\hline $\begin{array}{l}\text { Home } \\
\text { address }\end{array}$ & $\begin{array}{l}\text { Care of } \\
\text { address }\end{array}$ & Protocol & Port & Lifetime \\
\hline 3ffe::a:b:c:d & $3 f f c:: 1: 5: a: b: c: d$ & -1 & -1 & 150 \\
\hline 3ffe::a:b:c:d & $3 f f c:: 1: 6: a: b: c: a$ & 6 & 6935 & 200 \\
\hline $3 f f e:: a: b: c: d$ & $3 f f c:: 1: a: a: b: c: d$ & 17 & 7830 & 150 \\
\hline
\end{tabular}

By sending the RNL values embedded in the BU messages' headers, the home agent will have the knowledge of all mobile nodes' RNL values for each 
wireless access network. It can thus perform globally optimized access network selection on behalf of them.

If the procedure optimizing resources on the heterogeneous wireless network level turns out to decide to move a certain flow from one wireless access network to another, the home agent reflects that in its subsequent BAck messages sent to that mobile node. Also, the home agent reflects the move of that particular flow in its actual routing of packets associated with the flow.

\section{Optimization Procedure}

This section describes the optimized access network selection algorithm that the home agent may take on for newly arrived flows. The general idea is to allow the home agent to distribute such flows among available wireless access networks to achieve balanced loads between the wireless access networks.

This is an approximate solution to the bin packing problem [34], being a wellknown NP-hard optimization problem. A bin packing problem is called on-line if every item is packed without information on subsequent items, while an offline problem allows decisions to be taken with full knowledge of all the items. The model of on-line bin packing is used for assigning newly arrived flows to the best wireless access network while minimizing the sum of RNL values for all flows in the heterogeneous wireless network.

Formally, the access network selection (ANS) problem statement is defined as follows:

ANS Problem Statement: Given a function $a$ mapping a flow $f_{i}$ to access network $A_{j}$, i.e. $a\left(f_{i}\right)=A_{j}$ if $f_{i}$ is assigned to $A_{j}$, given a set of flows $F=f_{1}, \ldots, f_{n}$ and a fixed set of access networks $\underline{A}=A_{1}, \ldots, A_{m}$, find the assignments of the flows $F$ to the access networks $A$ that minimize $\sum_{i=1}^{n} R N L\left(a\left(f_{i}\right)\right)$.

The algorithm for the mobile node assigning a wireless access network to an outgoing flow is as follows:

(i) The mobile node measures RNL on each access network according to equations (1)-(5) in Section 3

(ii) The mobile node chooses the access network with the lowest RNL value for the flow and indicates the flow in the mobility signaling to the home agent (i.e. flow mobility option) along with the RNL value according to Section 3. If the mobile node does not accept the flow to be moved by the home agent to another wireless access network, the $\mathrm{R}$ flag is set

(iii) If the $\mathrm{R}$ flag is not set, the home agent may perform global optimization and decide to move the newly arrived flow to another wireless access network that the mobile node is connected to in order to achieve load balancing. Any move is indicated to the mobile node in the mobility signaling (BAck) and also reflected in the actual routing of packets associated with that particular flow

Incoming traffic is handled in the following way:

(i) The home agent looks in the binding cache if there is already an existing binding for that flow. If that is the case, that binding is used to route packets associated with the flow

(ii) Otherwise, the home agent looks for the default route (marked by "-1" in the mobility signaling) for the destination mobile node. The home 
agent considers using the default route for the flow, but may perform global optimization and choose to decide to use another wireless access network depending on the outcome of the optimization procedure. If such decision is taken, a binding in stored in the binding cache of the home agent, so that subsequent packets are routed the same way

\section{$5 \quad$ Simulations}

A scenario with a heterogeneous wireless network consisting of an LTE network and an IEEE 802.11g network with overlapping coverage areas was simulated. Values on delay and jitter were derived from simulations in OPNET Modeler [35] (figure 6).

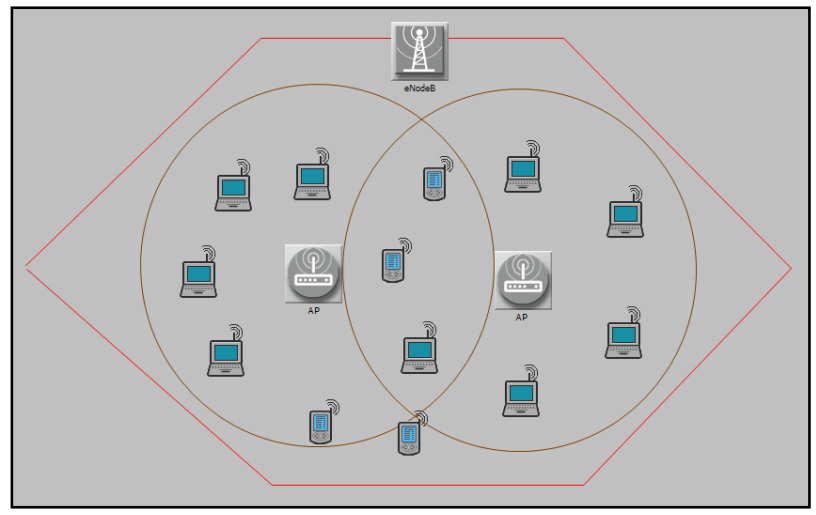

Fig. 6 Simulation model in OPNET Modeler

Three types of mobile nodes were used in the simulations:

1) Multimode terminals equipped with LTE and IEEE $802.11 \mathrm{~g}$ radio access technologies

2) LTE single mode terminals

3) IEEE $802.11 \mathrm{~g}$ single mode terminals

In the simulations mobile nodes of type 1 did not use the option to ask the home agent to retain a flow on a specific access network.

Two types of flows were used in the simulations:

1) Voice over IP (VoIP) calls (G.729A codec; $8 \mathrm{~kb} / \mathrm{s}, 100$ packets per second)

2) General IP flow (TCP-based file retrieval, $100 \mathrm{kB} / \mathrm{s}$ at the application layer)

Any mobile node could establish voice calls or general IP flows with a uniform distribution. All voice calls and general IP flows were destined to a server in the wired network.

Voice calls were arriving according to a Poisson process with the mean duration of 3 minutes distributed exponentially. Silence suppression was implemented using a Markov ON/OFF model with talk spurts exponentially distributed with a mean of 20 seconds and periods of silence exponentially distributed with a mean of 10 seconds. General IP flows were sent according to a Poisson process with the mean duration of 5 minutes distributed exponentially.

RNL values for each radio access technology and the numbers of ongoing VoIP calls and general IP flows respectively were calculated using output from OPNET Modeler. The constants were set so that $c=1$ and $h=1$. The LTE 
network used a FDD (Frequency Division Duplex) configuration with $3 \mathrm{MHz}$ of bandwidth allocated in both directions, while the data rate in the WLAN network was 54 Mbps.

Simulations were then performed using tailor-made software with arrival rates of $\lambda=\{0.5,1,1.5,2,2.5,3,3.5,4,4.5,5\}$ users/minute. 100 simulations for each arrival rate were performed. Furthermore, the simulations lasted for 1800 seconds, but results were collected only during the last 900 seconds. Weighted means of RNL for each wireless access network and the fraction of VoIP calls with MOS (Mean Opinion Score) less than 2.0 were collected.

\section{$6 \quad$ Results}

Three strategies for access network selection decision making were evaluated:

1) "WLAN if coverage" meaning that the mobile node decides to use WLAN if it is within the coverage area of any WLAN Access Point

2) "Local RNL-based" meaning that the mobile node decides to use the wireless access network with the lowest RNL value basing its decisions on locally calculated information only

3) "Globally optimized RNL-based" meaning that the mobile node measures the RNL values in all available wireless access networks, sends the values to the home agent, and allows the home agent to decide which wireless access network to assign new flows to

Below, RNL values for WLAN and LTE obtained from OPNET Modeler are shown (figure 7 and figure 8 respectively).

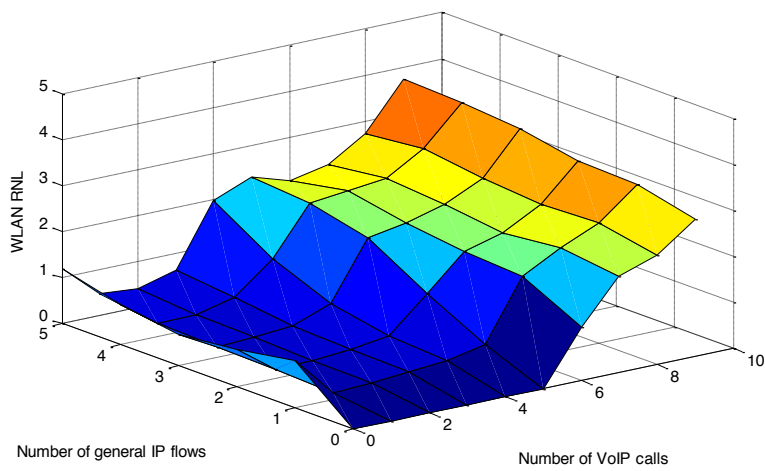

Fig. 7 RNL values for WLAN plotted against number of VoIP calls and number of general IP flows

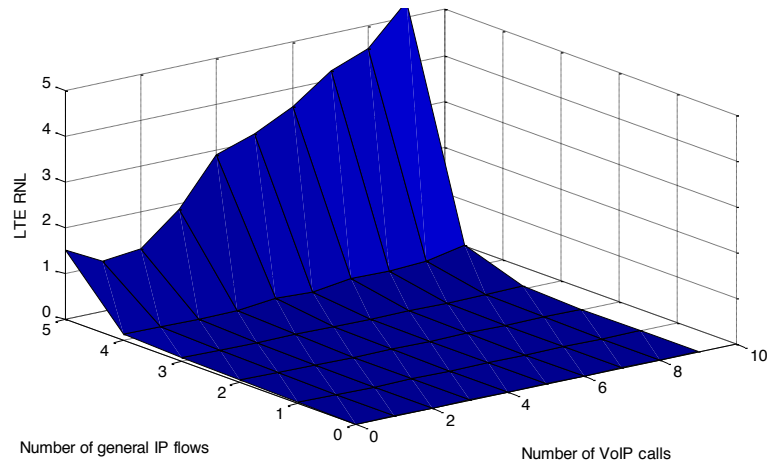

Fig. 8 RNL values for LTE plotted against number of VoIP calls and number of general IP flows

It was also noted that RNL values for LTE were higher for more general IP flows than 5 . 
The three strategies were then compared. The fractions of VoIP calls allocated to WLAN are plotted against arrival rate $(\lambda)$ (figure 9).

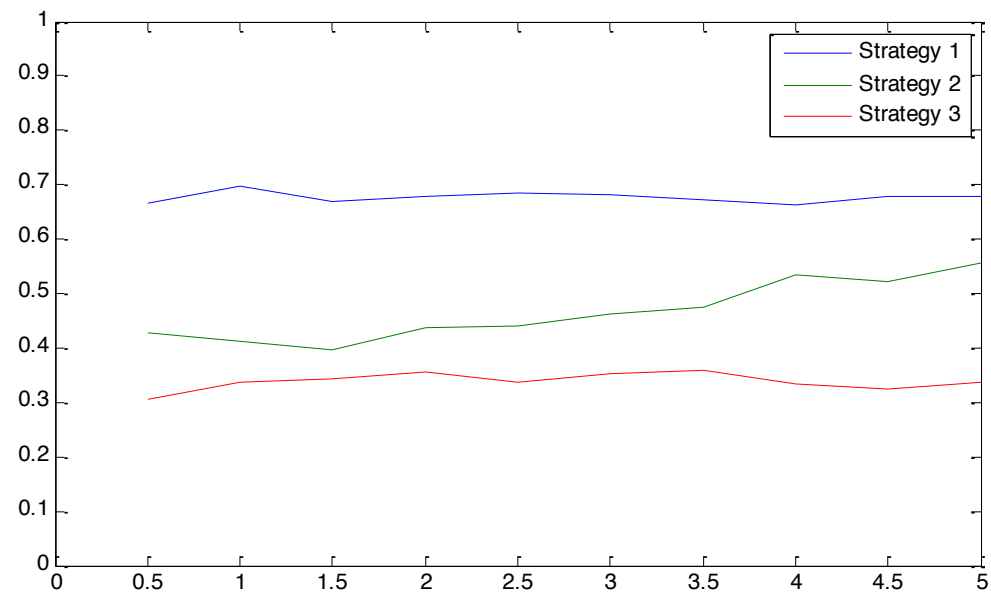

Fig. 9 Fraction of VoIP calls allocated to WLAN for each strategy plotted against arrival rate

Also, the fractions of VoIP calls with MOS below 2.0 are plotted against arrival rate $(\lambda)$ (figure 10 ).

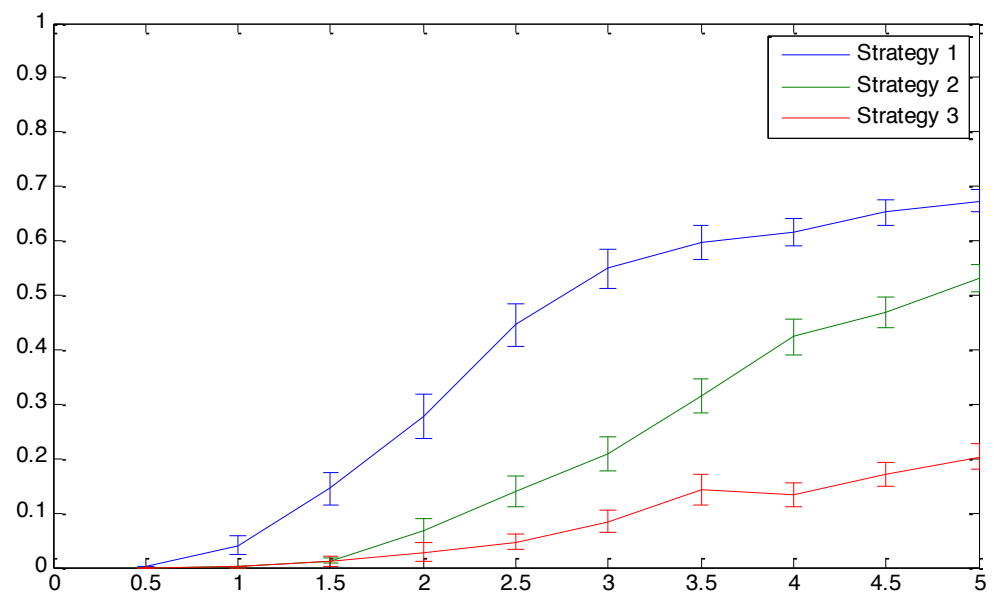

Fig. 10 Performance for each strategy in terms of fraction of VoIP calls with MOS below 2.0 plotted against arrival rate

The results depicted in figure 9 clearly show ${ }^{1}$ that using RNL as input for access network selection decisions outperforms the "WLAN if coverage" type of mechanism used in many handsets today. In addition, allowing the home agent to globally optimize the allocation of newly arrived flows to different wireless access networks improves the overall performance even further.

\section{$7 \quad$ Conclusions and Future Work}

The rationale behind using delay and jitter for computing the RNL metric is based on the fact that the higher the network load, the higher delay and jitter values will be experienced by any mobile node connected to that wireless access network. This is the case because WLAN and LTE use a shared channel where all flows compete for a common resource and leads to the RNL metric reflecting the network load in each wireless access network regardless if the mobile nodes are connected to the mobile overlay network or not. Furthermore, the signaling

\footnotetext{
${ }^{1}$ error bars indicate standard errors of the mean at $95 \%$ confidence level
} 
overhead in our proposal is minimal since existing mobility signaling, i.e. the BU messages and BAcks of the Mobile IPv6 protocol, is reused.

When comparing the solution presented in this article with related work [20-29] the most important difference is that our proposal is not using access technology-specific parameters of the wireless access networks like maximum capacity, typical SNR levels, etc. Our solution is lightweight and easy to deploy stepwise.

Future work includes integrating the model presented in this article with the proposed extension to IEEE 802.21 being presented in [36]. We also intend to integrate some of the functionality provided by the 3GPP ANDSF entity and the current 3GPP initiative standardizing IP Flow Mobility and seamless WLAN offload into our architecture.

Acknowledgments The work presented in this article is based on results from the HybriNet@Skellefteå, Mobile \& Open Service Access, and BasicNET projects supported by Skellefteå Kraft, EU structural funds (Objective 2), and VINNOVA respectively.

\section{References}

1. Perkins, C. (ed.) (2002). IP Mobility Support for IPv4, IETF, RFC 3344.

2. Johnson, D. B., Perkins, C. E., \& Arkko, J. (2004). Mobility support in IPv6. IETF, RFC 3775 .

3. Koh, S. J., Chang, M. J., \& Lee, M. (2004). mSCTP for Soft Handover in Transport Layer. IEEE Communications Letters, 8(3), 189-191.

4. Schulzrinne, H. \& Wedlund, E. (2000). Application-layer mobility using SIP. SIGMOBILE Mob. Comput. Commun. Rev. 4(3), 47-57.

5. Gundavelli, S. (ed.) (2008). Proxy Mobile IPv6, IETF, RFC 5213.

6. IEEE: IEEE 802.21: Local and metropolitan area networks: Media independent handover services (January 2009)

7. 3GPP TS 23.402, Architecture enhancements for non-3GPP accesses, V9.4.0 (March 2010)

8. 3GPP TS 24.302, Access to the 3GPP Evolved Packet Core (EPC) via non-3GPP access networks, V9.2.0 (March 2010)

9. Melia, T. (ed.), Bajko, G., Das, S., Golmie, N., \& Zuniga, JC. (2009). IEEE 802.21 Mobility Services Framework Design (MSFD). IETF, RFC 5677.

10. Bajko, G., \& Das, S. (2009). Dynamic Host Configuration Protocol (DHCPv4 and DHCPv6) Options for IEEE 802.21 Mobility Services (MoS) Discovery. IETF, RFC 5678.

11. Bajko, G. (2009). Locating IEEE 802.21 Mobility Services Using DNS. IETF, RFC 5679.

12. Open Mobile Alliance, OMA Device Management Protocol, www.openmobilealliance.org (February 2007)

13. 3GPP TS 24.312, Access Network Discovery and Selection Function (ANDSF) Management Object (MO), V9.1.0 (March 2010)

14. Das, S., \& Bajko, G., Dynamic Host Configuration Protocol (DHCPv4 and DHCPv6) Options for Access Network Discovery and Selection Function (ANDSF) Discovery, Internet Draft, draft-das-mipshop-andsf-dhcp-options-04.txt (February 2010)

15. 3GPP TS 23.261, IP Flow Mobility and seamless WLAN offload, V10.0 (June 2010)

16. Soliman, H. (ed.) (2009). Mobile IPv6 Support for Dual Stack Hosts and Routers, IETF, RFC 5555.

17. Wakikawa, R. (ed.), Devarapalli, V., Tsirtsis, G., Ernst, T., \& Nagami, K. (2009). Multiple Care-of Addresses Registration, IETF, RFC 5648.

18. Tsirtsis, G., Soliman, H., Montavont, N., Giaretta, G., \& Kuladinithi, K., Flow Bindings in Mobile IPv6 and NEMO Basic Support, Internet Draft, draft-ietf-mext-flow-binding10.txt (September 2010)

19. Tsirtsis, G., Giaretta, G., Soliman, H., \& Montavont N., Traffic Selectors for Flow Bindings, Internet Draft, draft-ietf-mext-binary-ts-04.txt (February 2010)

20. McNair, J. \& Fang Zhu (2004). Vertical handoffs in fourth-generation multinetwork environments. IEEE Wireless Communications, 11(3), 8-15. 
21. Gazis, V., Alonistioti, N., \& Merakos, L. (2005). Toward a generic "always best connected" capability in integrated WLAN/UMTS cellular mobile networks (and beyond). IEEE Wireless Communications, 12(3), 20-29.

22. Song, Q. \& Jamalipour, A. (2005). Network selection in an integrated wireless LAN and UMTS environment using mathematical modeling and computing techniques. IEEE Wireless Communications, 12(3), 42-48.

23. Xing, B. \& Venkatasubramanian, N. (2005). Multi-constraint dynamic access selection in always best connected networks. In MobiQuitous 2005, San Diego, CA, USA.

24. Haydar, J., Ibrahim, A., \& Pujolle, G. (2008). A new access selection strategy in heterogeneous wireless networks based on traffic distribution, In Wireless Days 2008, Dubai, United Arab Emirates.

25. Gustafsson, E. \& Jonsson, A. (2003). Always best connected. IEEE Wireless Communications, 10(1), 49-55.

26. Perera, E., Boreli, R., Herborn, S., Georgiades, M., Eisl, J., \& Hepworth, E. (2008). A mobility toolbox architecture for all-IP networks: an ambient networks approach. IEEE Wireless Communications, 15(2), 8-16.

27. Kong, K., Lee, W., Han, Y., Shin, M., \& You, H. (2008). Mobility Management for AllIP Mobile Networks: Mobile IPv6 vs. Proxy Mobile IPv6. IEEE Wireless Communications, 15(2), $36-45$.

28. Kafle, V. P. \& Inoue, M. (2010). Locator ID Separation for Mobility Management in the New Generation Network. Journal of Wireless Mobile Networks, Ubiquitous Computing, and Dependable Applications, 1(2/3), 3-15.

29. Kiriyama, S., Wakikawa, R., Xia, J., \& Teraoka, F. (2010). Context Reflector for Proxy Mobile IPv6, Journal of Wireless Mobile Networks, Ubiquitous Computing, and Dependable Applications, 1(2/3), 36-51.

30. Lee, K., Rhee, I., Lee, J., Yi, Y., \& Chong, S. (2010). Mobile Data Offloading: How Much Can WiFi Deliver? In ACM SIGCOMM 2010, New Delhi, India.

31. Åhlund, C., Brännström, R., \& Zaslavsky, A. (2006). Traffic load metrics for multihomed mobile IP and global connectivity. Telecommunication Systems, 33(1-3), 155-185.

32. Brännström, R., Åhlund, C., Andersson, K., \& Granlund, D. (2007). Multimedia Flow Mobility in Heterogeneous Networks using Multihomed Mobile IP. Journal of Mobile Multimedia, 3(3), 218-234.

33. Åhlund, C., Wallin, S., Andersson, K., \& Brännström, R. (2008). A service level model and Internet mobility monitor. Telecommunication Systems, 37(1), 49-70.

34. Coffman, E. G., Csirik, J., \& Woeginger, G. J. (2002). Approximation Solutions to Bin Packing Problems. Handbooks of Applied Optimization, Oxford University Press, Oxford

35. OPNET Modeler 16.0.A PL4, www.opnet.com

36. Andersson, K, Forte, A. G., \& Schulzrinne, H. (2010). Enhanced Mobility Support for Roaming Users: Extending the IEEE 802.21 Information Service. In WWIC 2010, LNCS $6074,52-63$.

\section{Author Biographies}

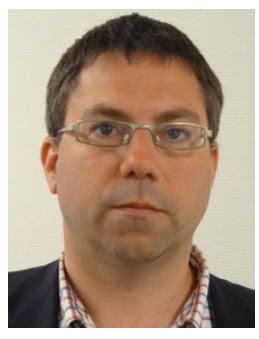

Karl Andersson (karl.andersson@1tu.se) received his M.Sc. degree in computer science and technology from Royal Institute of Technology, Stockholm, Sweden, in 1993. After spending more than 10 years as an IT consultant working mainly with telecom clients he returned to academia and earned his Ph.D. degree from Luleå University of Technology (LTU) in 2010 in Mobile Systems. Following his Ph.D. degree Karl was appointed Assistant Professor of Pervasive and Mobile Computing at LTU in 2011. Currently Karl is also a postdoctoral research fellow at Internet Real-Time Laboratory, Columbia University, New York, USA. His research interests are centered around mobility management in heterogeneous networking environments, mobile e-services, and locationbased services. Karl is a senior member of the IEEE. 


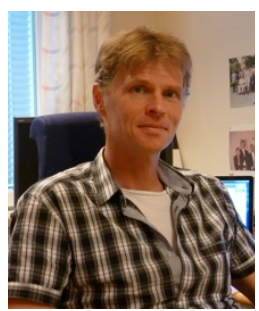

Christer Åhlund (christer.ahlund@ltu.se) is Chaired Professor of Pervasive and Mobile Computing at Luleå University of Technology (LTU). He received his M.Sc. degree in Computer Science from Uppsala University and his Ph.D. degree from LTU. Christer is currently head of the division of Computer Science at LTU and his research deals with IP mobility, ad-hoc networks, heterogeneous access networks and media distribution. He has been working in the industry for 10 years as a programmer, systems engineer and project manager. Christer is a senior member of the IEEE. 